\title{
ÁCIDOS GRAXOS E O SISTEMA CARDIOVASCULAR
}

\section{Priscilla Regina Nasciutti ${ }^{1}$, Ana Paula Araújo Costa ${ }^{1}$, Marcelo Borges dos Santos Júnior $^{2}$, Nayara Gonçalves de Melo ${ }^{3}$, Rosângela de Oliveira Alves Carvalho ${ }^{4}$ \\ ${ }^{1}$ Doutoranda em Ciência Animal, Escola de Veterinária e Zootecnia da Universidade Federal de Goiás, Goiás, Brasil (prinasciutti@gmail.com) \\ ${ }^{2}$ Mestre em Ciência Animal, Médico Veterinário Autônomo, Goiás, Brasil \\ ${ }^{3}$ Graduanda de Medicina Veterinária, Escola de Veterinária e Zootecnia da Universidade Federal de Goiás, Goiás, Brasil \\ ${ }^{4}$ Professor Doutor, Escola de Veterinária e Zootecnia da Universidade Federal de Goiás, Goiás, Brasil}

\author{
Recebido em: 08/09/2015 - Aprovado em: 14/11/2015 - Publicado em: 01/12/2015 \\ DOI: http://dx.doi.org/10.18677/Enciclopedia_Biosfera_2015_066
}

\begin{abstract}
RESUMO
Os ácidos graxos são substâncias que fazem parte da constituição das moléculas de lipídeos e podem ser classificados segundo a sua estrutura química em ácidos graxos saturados e insaturados. A ingestão de lipídeos na dieta constitui atualmente em um dos fatores de risco para o desenvolvimento de doenças cardiovasculares, classificadas como a principal causa de morte nos dias de hoje. Dietas ricas em ácidos graxos saturados e insaturados trans são responsáveis pelo desenvolvimento de hipercolesterolemia, por isso o consumo está relacionado ao aumento do risco de desenvolvimento de doenças cardiovasculares. As pesquisas envolvendo ácidos graxos insaturados da série $\omega-3$, mostram que em humanos, os ácidos graxos desta classe promovem efeitos cardioprotetores envolvendo menor processo inflamatório, prevenção à arritmias, vasodilatação e controle nos níveis de lipídeos no sangue. Portanto, o objetivo deste artigo é apresentar uma visão geral a respeito dos ácidos graxos e seus efeitos sobre o sistema cardiovascular.
\end{abstract}

PALAVRAS-CHAVE: antiarrítmico, coração, deslipidemias, lipídeos, ômega-3

\section{FATTY ACID AND CARDIOVASCULAR SYSTEM}

\begin{abstract}
Fatty acids are substances that are part of the constitution of lipid molecules and can be classified according to their chemical structure in saturated and unsaturated fatty acids. The ingestion of lipids represent one of the risk factors for developement of cardiovascular disease, classified as a major cause of death nowadays. Diets rich in saturated and trans-unsaturated fatty acids are responsible for the development of hypercholesterolemia, so their consumption is associated with an increased risk of developing cardiovascular diseases. Research involving unsaturated fatty acids of the $\omega-3$ series, show that in humans, the fatty acids of this class promote cardioprotective effects by decreasing inflammatory process, preventing of
\end{abstract}


arrhythmias, vasodilation and controling the levels of lipids in the blood. Therefore, the purpose of this article is to present an overview about the fatty acids and their effects on the cardiovascular system.

KEYWORDS: antiarrhythmic, dyslipidemias, heart, lipids, omega-3

\section{INTRODUÇÃO}

As doenças cardiovasculares são atualmente a principal causa de mortes no mundo, sendo responsáveis por $30 \%$ delas. Acredita-se que em 2030, o número de pessoas que morrerão vítimas de doenças cardíacas e de acidente vascular cerebral atingirão 23,3 milhões, e que as doenças cardiovasculares continuarão a ser a principal causa de morte. A maioria dessas doenças pode ser prevenida, diminuindo-se fatores de risco associados, como por exemplo, o consumo de lipídeos na dieta (WHO, 2013).

Os lipídeos, popularmente chamados de gorduras, são compostos insolúveis em água constituídos de uma série de substâncias, entre elas os ácidos graxos (NELSON \& COX, 2011). Os ácidos graxos são ácidos carboxílicos com cadeias hidrocarbonadas, classificados segundo o comprimento da cadeia de carbonos (cadeia curta, média e longa), a presença e número de duplas ligações (saturados e insaturados) e a configuração das duplas ligações (cis e trans) (SANTOS et al., 2013).

Os ácidos graxos saturados (AGS) são assim denominados por não conterem dupla ligação em sua estrutura. São obtidos de algumas gorduras de origem animal e vegetal (MOREIRA et al., 2002). Muitas pesquisas indicam que dietas ricas em AGS promovem hipercolesterolemia (SANTOS et al., 2012), e por isso o consumo está relacionado ao aumento do risco de desenvolvimento de doenças cardiovasculares (SANTOS et al., 2013).

Os ácidos graxos insaturados (AGI) são aqueles que contêm uma ou mais duplas ligações na cadeia hidrocarbonada. Podem ser monoinsaturados ou poliinsaturados (RAPOSO, 2010) e pertencem a diferentes séries, sendo classificados em $\omega-3, \omega-6$ e $\omega-9$ (CASA NOVA \& MEDEIROS, 2011).

Os ácidos graxos trans (AGT) são isômeros geométricos dos ácidos graxos insaturados (AGI), formados a partir do processo de bio-hidrogenação de gorduras de origem animal e de hidrogenação parcial ou total de óleos vegetais ou marinhos (BERTOLINO et al., 2006). Assim como observado com os AGS, os AGT têm ação hipercolesterolêmica (SANTOS et al., 2012) e podem alterar a agregação plaquetária e a função vascular gerando aumento do risco de doenças cardiovasculares (NELSON \& COX, 2011).

Os $A G I$ da série $\omega-9$ são monoinstaturados, encontrados nos óleos de origem vegetal. Quando comparados a dietas ricas em AGS proporcionam menores alterações no perfil lipídico (KASTORINI et al., 2011) e pressão arterial (ALONSO et al., 2006). Os AGI da série $\omega-6$ são poli-insaturados, encontrados em castanhas, sementes e óleos vegetais (RAPOSO, 2010). Os ácidos graxos pertencentes a esta série são precursores de mediadores pró-inflamatórios que promovem vasoconstrição e ação pró-agregatória (LOTTENBERG, 2009), entretanto, os estudos relacionados ao sistema cardiovascular são controversos (SANTOS et al., 2013).

Os AGI da série $\omega-3$ também poli-insaturados, são encontrados nos peixes (RAPOSO, 2010). Ao contrário dos resultados controversos em relação aos AGI da série $\omega-6$, as pesquisas mostram que em humanos, os ácidos graxos $\omega-3$ promovem efeitos antiinflamatórios, antiarrítmicos, vasodilatadores, atuando também 
no controle das dislipidemias e melhora da hemodinâmica vascular (CASA NOVA \& MEDEIROS, 2011; MOZAFFARIAN \& WU, 2011).

Desta forma, o objetivo deste artigo é apresentar uma visão geral a respeito dos ácidos graxos, bem como enfatizar seus efeitos sobre o sistema cardiovascular, dando especial atenção aos ácidos graxos $\omega-3$, responsáveis por promover efeitos benéficos aos organismos vivos.

\section{ÁCIDOS GRAXOS: CONCEITO, CLASSIFICAÇÃO E NOMENCLATURA}

Os lipídeos, popularmente chamados de gorduras, são compostos constituídos de uma série de substâncias que desempenham diferentes papéis no organismo e que tem como característica principal a insolubilidade em água. Os componentes lipídicos estão presentes em diferentes estruturas dos organismos vivos (membranas celulares e tecidos) e atuam principalmente como reserva de energia, cofatores enzimáticos, transportadores de elétrons, âncoras hidrofóbicas, agentes emulsificantes, hormônios e mensageiros intracelulares (NELSON \& COX, 2011).

Os lipídeos podem ser classificados em lipídeos compostos, formados basicamente por moléculas de glicerol e de ácidos graxos (ligados ou não a aminoálcoois) ou lipídeos simples, que não produzem ácidos graxos após o processo de hidrólise. Os triglicerídeos são os lipídeos compostos mais abundantes no organismo e que tem a função de armazenamento de energia; os fosfoglicerídeos e os esfingolipídeos são outros exemplos de lipídeos compostos, constituintes das membranas celulares. Entre os lipídeos simples, encontram-se os esteróis, sendo o colesterol o mais importante deles, além dos derivados dos ácidos graxos com função metabólica e as vitaminas lipossolúveis (A, D, E e K) (GONZÁLEZ, 2006). Alguns lipídeos são sintetizados pelos organismos vivos, outros são obtidos por meio da alimentação. Os lipídeos da dieta são encontrados em fontes animais e vegetais, incluindo os de vida marinha (MOREIRA et al., 2002).

Os ácidos graxos são ácidos carboxílicos, geralmente monocarboxílicos, com cadeias hidrocarbônicas, representadas pela forma $\mathrm{RCO}_{2} \mathrm{H}$. O grupamento $\mathrm{R}$ é uma cadeia de carbonos longa, não ramificada, com número par de átomos de carbono, podendo ser saturada (ligações simples) ou conter uma ou mais duplas ligações (insaturações). $O$ grupo carboxila constitui a região polar e a cadeia $R$ a região apolar da molécula. São classificados segundo o comprimento da cadeia de carbonos, a presença e o número de duplas ligações e a configuração das duplas ligações (SANTOS et al., 2013).

Com relação ao comprimento da cadeia hidrocarbônica, são classificados como cadeia curta (quatro a oito átomos de carbono), cadeia média (oito a 12 átomos de carbono) e cadeia longa (mais de 12 átomos de carbono). Para a presença de duplas ligações são classificados em saturados (não possuem dupla ligação entre os átomos de carbono) ou insaturados (possuem uma ou mais duplas ligações dentro da cadeia de carbonos). Dentre os ácidos graxos insaturados, os monoinsaturados possuem apenas uma dupla ligação e os poli-insaturados contém duas ou mais duplas ligações e ainda podem ter configurações cis e trans. A maioria dos ácidos graxos possui cadeias não ramificadas (lineares), entretanto alguns de ocorrência rara são ramificados, e outros contêm estruturas cíclicas ligadas à cadeia hidrocarbônica (RAPOSO, 2010).

A nomenclatura utilizada para os ácidos graxos pode ser derivada da fonte de origem do ácido como, por exemplo, ácido palmítico, originado do óleo de 
palma ou por um nome sistemático baseado em sua estrutura química. O nome sistemático é iniciado pela palavra "ácido" seguido de um prefixo referente ao número de átomos de carbono da maior cadeia hidrocarbônica que contenha o grupo carboxila e o sufixo "óico". Os átomos de carbono podem ser numerados a partir do grupo carboxila (numeração delta - $\Delta$ ou letras gregas) ou a partir do carbono mais distante da carboxila (numeração $n$ ou ômega - $\omega$ ). Uma nomenclatura simplificada também pode ser utilizada, em que se especifica 0 comprimento da cadeia hidrocarbônica (número de carbonos) separado por dois pontos, seguido do número de instauração (por exemplo, ácido oleico com 18 carbonos e uma dupla ligação - 18:1) (NELSON \& COX, 2011).

\section{ÁCIDOS GRAXOS SATURADOS}

Os ácidos graxos saturados (AGS) são ácidos graxos sem insaturações ou duplas ligações em sua cadeia hidrocarbônica (MOREIRA et al., 2002). São obtidos na dieta a partir de gorduras de origem animal (carnes, leite e derivados) e vegetal (coco e cacau). Basicamente, os ácidos graxos desta classe são de cadeia média e de cadeia longa A maioria dos AGS é de cadeia longa. São exemplos, o mirístico (14:0), encontrado no leite e seus derivados; o palmítico (16:0), cujas principais fontes são a gordura animal e o óleo de palma; e o esteárico (18:0), presente na gordura do cacau. Em temperatura ambiente, esses AGS de cadeia longa encontram-se no estado sólido (LOTTENBERG, 2009).

Quando ingeridos, os AGS de cadeia média são absorvidos na forma não esterificada e transportados ao fígado, ligados a albumina, onde são rapidamente metabolizados. Já os AGS de cadeia longa, sofrem um processo de esterificação, formando os triglicerídeos, que podem permanecer na corrente sanguínea, transportados por quilomícrons, ou ser liberados para os tecidos, atuando como forma de armazenamento de gordura no organismo (SANTOS et al., 2013).

\section{Efeitos dos ácidos graxos saturados sobre o sistema cardiovascular}

Estudo realizado na década de 1980 para avaliar a influência da ingestão de AGS comprovou que dietas ricas neste tipo de ácido graxo promovem aumentos nas concentrações de colesterol total e das lipoproteínas de baixa densidade (LDL) no plasma, quando comparadas a dietas livres de gordura e colesterol, por isso, 0 consumo deste tipo de ácido graxo é relacionado com o aumento do risco de desenvolvimento de doenças cardiovasculares (SANTOS et al., 2013).

O colesterol é uma molécula essencial para a formação de membranas celulares e para a síntese de ácidos biliares e hormônios esteroidais. Pode ser obtido a partir da síntese endógena, ou por meio da alimentação, e o transporte no organismo é realizado por lipoproteínas (GONZÁLES, 2006).

As lipoproteínas são a associação de lipídeos com proteínas. As LDL são a principal transportadora de colesterol e triglicerídeos endógenos do fígado para os tecidos, por meio da ligação à receptores na superfície celular, que fazem com que esses componentes sejam captados pelas células por endocitose. Já as lipoproteínas de alta densidade (HDL), transportam o colesterol endógeno dos tecidos para o fígado e é o único capaz de eliminar grandes quantidades de colesterol plasmático. Ainda existem as lipoproteínas de densidade muito baixa (VLDL) e as de densidade intermediária (IDL). O excesso de LDL no plasma é atualmente considerado um dos principais fatores de risco para o desenvolvimento de aterosclerose, pois são oxidadas e captadas por macrófagos que habitam as 
placas ateroscleróticas, auxiliando no processo de acúmulo de lipídeos e formação de placas calcificadas fibrosas da musculatura lisa da parede interna dos vasos. Essas lesões podem reduzir a luz do vaso e ainda ocluí-los, promovendo a parada do fluxo sanguíneo e o infarto da região (VOET \& VOET, 2013).

Entre os AGS, o aumento dos níveis de colesterol no sangue é observado principalmente na ingestão do ácido palmítico e do ácido mirístico, entretanto o ácido esteárico não promove hipercolesterolemia, pois é rapidamente convertido em ácido oleico (ácido graxo insaturado) no fígado (LOTTENBERG, 2009).

Em busca de determinar fatores de risco para doenças cardiovasculares, em 1996 um estudo conduzido na Finlândia já demonstrava que reduções no conteúdo total de gordura da dieta (38\% para $34 \%$ ) e na quantidade de gordura saturada ( $21 \%$ para $16 \%$ ) foram capazes de reduzir em $16 \%$ os níveis de colesterol total. Apesar de outros fatores positivos terem auxiliado nesta redução (maior ingestão de frutas e vegetais e aumento na ingestão de ácidos graxos poliinsaturados), concluiu-se que os AGS são responsáveis pela hipercolesterolemia (PIETINEN et al., 1996).

Segundo a Sociedade Brasileira de Cardiologia, a base fisiológica para os efeitos prejudiciais dos AGS ao sistema cardiovascular é a aterosclerose, pois esta se associa diretamente com fatores de risco como a hipercolesterolemia. A influência que os AGS possam ter sobre parâmetros como a pressão arterial sistêmica e a concentração de biomarcadores inflamatórios ainda é controversa (SANTOS et al., 2013).

Estudo recente realizado por EBBESSON et al. (2015) em uma população de ocidentais nativos do Alasca mostram que as altas concentrações de gorduras saturadas no sangue são prejudiciais para a saúde cardiovascular quando comparados a alguns ácidos graxos insaturados, que podem ter efeitos nulos e até protetores. O estudo em questão associou as concentrações plasmáticas dos ácidos graxos saturados com fatores de risco para o desenvolvimento de doenças cardiovasculares, como aumento de triglicérides, pressão arterial, frequência cardíaca, índice de massa corporal e glicemia em jejum.

Entretanto, MICHA \& MEZAFFARIAN (2010) ressaltaram que os efeitos dos AGS podem ser controversos, pois a maioria dos estudos compara os AGS a outros nutrientes substitutivos. Também é possível verificar que os efeitos no perfil lipídico são diferentes, a depender de qual AGS é estudado. Quando se substitui os AGS por carboidratos, por exemplo, é possível verificar níveis maiores de colesterol total, LDL e HDL. Nesta mesma situação, o ácido láurico (12:0) é o responsável por maiores valores de LDL, seguido do ácido mirístico (14:0) e do ácido palmítico (16:0).

\section{ÁCIDOS GRAXOS INSATURADOS}

Os ácidos graxos insaturados (AGI) são aqueles que contêm uma ou mais duplas ligações em sua cadeia de carbonos. Os AGI são de dois tipos, monoinsaturados (AGMls), que possuem apenas uma dupla ligação e poliinsaturados (AGPIs) que contém duas ou mais duplas ligações (RAPOSO, 2010). Esses ácidos graxos pertencem a diferentes séries, que são definidos pela localização da primeira dupla ligação na cadeia de carbono a partir do grupo metil, identificados pela letra $\omega$. Com base nesta classificação, os principais AGI são classificados nas série $\omega-3, \omega-6$ e $\omega-9$ (CASA NOVA \& MEDEIROS, 2011). 


\section{Ácidos graxos trans}

Os ácidos graxos trans (AGT) são estruturalmente isômeros geométricos dos ácidos graxos insaturados (AGI). Estes ácidos resultam do processo de biohidrogenação de gorduras derivadas da carne e leite de animais ruminantes (este processo ocorre naturalmente por ação da microbiota do rúmen). Ainda, estão presentes em produtos alimentícios industrializados, que sofrem hidrogenação parcial ou total nos óleos vegetais ou de organismos marinhos (BROUWER et al., 2010). Estima-se que 80 a $90 \%$ de todos os AGT provenientes da dieta sejam obtidos de óleos vegetais parcialmente hidrogenados, encontrados em margarinas sólidas ou cremosas, bolachas e biscoitos, sorvetes, batatas fritas comerciais ou em qualquer outro alimento que contenha gordura vegetal hidrogenada (BERTOLINO et al., 2006).

O metabolismo dos AGT nos mamíferos se dá pela rápida absorção e incorporação aos tecidos, com concentrações que refletem a quantidade e conteúdo obtido por meio da dieta. Neste processo, os AGT passam a competir com o metabolismo de ácidos graxos essenciais, que não são sintetizados pelo organismo, promovendo inibição dos mesmos à incorporação na membrana fosfolipídica, alterando assim todo o processo de formação de precursores inflamatórios (MOREIRA et al., 2002).

\section{Efeitos dos ácidos graxos trans no sistema cardiovascular}

Assim como observado com os AGS, os AGT apresentam ação hipercolesterolêmica, promovendo aumento nos níveis de triglicerídeos e da LDL e diminuição nos níveis de HDL. Esses fatores fazem com que o risco de doenças cardiovasculares seja maior nos indivíduos que ingerem grandes quantidades deste tipo de ácidos graxos (NELSON \& COX, 2011).

Em estudo realizado por LEMAITRE et al. (2001) com pacientes humanos após parada cardíaca primária, sem histórico de doenças cardíacas anteriormente reconhecidas, concluiu que níveis elevados de AGT na membrana de eritrócitos aumentam o risco de parada cardíaca primária em 1,5 vezes. Neste estudo, não foi observada relação com a ingestão de ácido oleico trans (18:1), em contrapartida, o risco para tal evento aumenta para três vezes, quando relacionado com a ingestão de ácido linoleico trans (18:2).

Após alguns anos, LEMAITRE et al. (2006) realizaram estudos com pacientes em idade mais avançada, com grupos com e sem doenças cardiovasculares diagnosticadas. Os resultados obtidos foram semelhantes ao do estudo anterior, enfatizando um aumento de quatro vezes no risco de doença cardíaca isquêmica quando se encontram níveis elevados de ácido linoleico trans (18:2) na membrana de eritrócitos, concluindo que a classe de ácido graxo trans em questão altera o risco de desenvolvimento de doenças cardíacas isquêmicas fatais e morte súbita.

SUN et al. (2007) realizaram estudo com mulheres e observaram que altos teores de AGT nas membranas dos eritrócitos se relacionam com o aumento em três vezes do risco de doenças coronarianas. Acredita-se que esse risco esteja ligado ao aumento da inflamação sistêmica, mediado pelos altos níveis de LDL e disfunção endotelial. MENTE et al. (2009) afirmaram que os níveis de AGT nas membranas dos eritrócitos e de outras células refletem os níveis do consumo 
alimentar, e que, portanto, a diminuição da quantidade de AGT ingeridos levaria a diminuição de AGT nas membranas celulares e, consequentemente, no risco de doenças cardiovasculares.

\section{Ácidos graxos monoinsaturados (AGMls)}

Os ácidos graxos monoinsaturados são caracterizados por conterem apenas uma instauração na cadeia hidrocarbônica. O principal representante dos AGMIs é o ácido oleico (18:1), classificado como AGl da série $\omega-9$, sendo o mais frequentemente encontrado na natureza. As principais fontes de ácido oleico são os óleos de oliva e canola, entretanto este pode ser sintetizado por todos os mamíferos. O ácido erúcico (22:1), também é obtido do óleo de canola, porém é eliminado no processo de extração do óleo devido ao sabor indesejável e por produzir miopatias nos seres humanos (LOTTENBERG, 2009). Além do azeite de oliva, os óleos de girassol, soja, cártamo, milho e amendoim também são fontes de ácido oleico (ALONSO et al., 2006).

Os estudos que relataram a influência dos AGMls sobre o sistema cardiovascular geralmente o fazem por meio de uma comparação com outros nutrientes. Quando se comparou a ingestão de uma dieta rica em AGMls com uma dieta hiperlipídica foi possível observar que a primeira resulta em menores valores de triglicerídeos e colesterol total e discretos aumentos nos valores de HDL (SANTOS et al., 2013).

KASTORINI et al. (2011) realizaram uma revisão sistemática de estudos epidemiológicos para avaliar os efeitos da dieta do Mediterrâneo, conhecida atualmente por ter efeitos cardioprotetores. Este padrão alimentar é caracterizado por um elevado consumo de ácidos graxos AGMls, por meio de azeitonas e azeite de oliva, associados com a ingestão diária de frutas, legumes, cereais, consumo semanal de peixe, pequena quantidade de produtos lácteos e de carne vermelha. Os resultados desta análise concluíram que esse padrão alimentar está associado com aumentos discretos nos níveis de HDL $(1,17 \mathrm{mg} / \mathrm{dL})$ e com reduções de $6,14 \mathrm{mg} / \mathrm{dL}$ de triglicerídeos.

Embora existam algumas inconsistências, as pesquisas relacionadas à influência da dieta do Mediterrâneo sobre a hipertensão arterial evidenciaram que a ingestão de AGMls é inversamente proporcional aos valores de pressão arterial, em pacientes humanos. Entretanto, esta resposta é somente observada diante da ingestão de azeite de oliva, rico em ácido oleico, e não em dietas ocidentais, onde as fontes de AGMls são alguns tipos de carne. Acredita-se que esta redução na pressão arterial, verificada com o azeite de oliva, seja mediada pela mudança na constituição dos ácidos graxos nas membranas celulares (ALONSO et al., 2006). Os estudos indicam ainda, no contexto da dieta do Mediterrâneo, que a ingestão de AGMIs está relacionada com menores riscos de desenvolvimento de doença arterial coronariana e morte (MENTE et al., 2009).

\section{Ácidos graxos poli-insaturados (AGPI)}

Os principais representantes dos AGPIs são os AGI da série $\omega-6$ e $\omega-3$. Os $A G I$ da série $\omega-6$ são encontrados em castanhas, sementes e óleos vegetais, com os de milho, girassol e soja (RAPOSO, 2010). O ácido linoleico (18:2) é um 
exemplo desta série e originará, no processo de metabolização, o ácido araquidônico (AA - 20:4) também da série $\omega-6$ (CASA NOVA \& MEDEIROS, 2011).

Os resultados de estudos que avaliaram a relação da ingestão de ácidos graxos $\omega-6$ e o risco de doenças cardíacas, principalmente as coronarianas (ácido linoleico e ácido araquidônico) mostraram resultados controversos. Apesar de serem precursores de mediadores pró-inflamatórios que promovem vasoconstrição e ação pró-agregatória e de aumentarem os níveis de LDL, quando comparados com AGS, eles ainda são capazes de reduzir o risco de doenças coronarianas (LOTTENBERG, 2009). Entretanto, quando comparada a ingestão de AGPIs $\omega-6$ com AGPIs $\omega-3$, o risco de doenças coronarianas é maior (MENTE et al., 2009).

Os AGI da série $\omega$-3 são encontrados nos peixes, principalmente savelha, salmão, atum e anchovas (RAPOSO, 2010). O ácido a-linolênico (18:3) é o principal representante desta série e é encontrado em plantas e animais marinhos. Após seu metabolismo, originará os ácidos eicosapentaenoico (EPA - 20:5) e docosahexaenoico (DHA - 22:6), que também podem ser encontrados em grandes concentrações nos óleos de peixes de águas frias e profundas, principalmente cavala, sardinha, salmão e truta (CASA NOVA \& MEDEIROS, 2011).

Os ácidos linoleico (18:2 $\omega$-6) e $\alpha$-linolênico (18:3 $\omega$-3) são essenciais aos mamíferos, pois suas células não tem a capacidade de inserir uma dupla ligação e sintetizá-los, por isso estes ácidos graxos devem ser obtidos pela alimentação (LOTTENBERG, 2009; COLUSSI et al., 2014).

Ao contrário dos resultados controversos observados nos estudos com os ácidos graxos $\omega-6$, as pesquisas em humanos e em animais com os ácidos graxos w-3 mostraram que são capazes de melhorar fatores hemodinâmicos cardíacos como a pressão arterial, enchimento diastólico do ventrículo esquerdo, frequência cardíaca e função endotelial. Além disso, também foram demonstrados efeitos antiarrítmicos, antinflamatórios e anti-ateroescleróticos, além de promoverem a redução de triglicérideos (ADKINS \& KELLEY, 2010).

\section{Metabolismo dos ácidos graxos essenciais (série $\omega-3$ e $\omega-6$ )}

Os ácidos graxos essenciais das séries $\omega-3$ e $\omega-6$ têm funções importantes relacionadas aos processos de coagulação e inflamação (LOTTENBERG, 2009). Estes ácidos graxos vão produzir metabólitos, denominados eicosanoides, que são responsáveis pela modulação da resposta inflamatória do organismo. O grupo dos eicosanoides é formado pelas prostaglandinas, prostaciclinas, tromboxanos, leucotrienos e derivados hidroxilados, com a principal função de regular a função celular (CASA NOVA \& MEDEIROS, 2011).

No processo de metabolização, o a-linolênico (ALA) é convertido em ácido eicosapentaenoico (EPA) e em ácido docosahexaenoico (DHA), já o ácido linoleico (LA) converte-se em ácido araquidônico (AA). Este processo é mediado pelas enzimas $\Delta 6$-desaturase, alongase e $\Delta 5$-desaturase e normalmente envolve as vias de metabolização das cicloxigenases (COX) e lipoxigenases (LOX) (CASA NOVA \& MEDEIROS, 2011).

O AA é preferencialmente metabolizado pela via das COXs e será precursor de prostanoides da classe 2 (prostaglandinas E2 (PGE2), tromboxanos A2 (TXA2) e prostaciclinas 2 (PCI2) e leucotrienos da classe B4 (LTB4), esses eicosanoides formados tem efeitos pró-inflamatórios, apresentando ação quimiotática, vasoconstritora e de agregação plaquetária. O AA também é precursor da síntese de epóxidos, agentes que são potentes vasoconstritores e responsáveis 
pelo aumento do depósito de cálcio nas células endoteliais. O EPA, preferencialmente degradado pela via LOX, levará a produção de prostanoides da classe 3 (prostaglandinas E3 - PGE3, tromboxanos A3 - TXA3 e prostaciclinas 3 PCI3) e leucotrienos da classe 5 (LTB5), que por sua vez apresentam ações antiinflamatórias e anti-agregação plaquetária. O DHA não é substrato para as vias COX e LOX, mas atua inibindo a síntese de eicosanoides a partir do $\omega$-6, inibindo, portanto, a liberação de AA da membrana (LOTTENBERG, 2009; RAPOSO, 2010).

Uma vez que são utilizadas as mesmas enzimas no processo de produção de eicosanoides, acredita-se que exista uma competição entre os ácidos graxos essenciais em sua entrada no processo de alongamento e dessaturação, que conduzirá à síntese das respectivas classes de eicosanoides. Sabe-se que as enzimas envolvidas no processo "preferem" o ALA, levando à produção de EPA e DHA, que bloqueiam a ação da enzima $\Delta 6$-dessaturase, inibindo a conversão do LA em AA e, consequentemente, a produção de eicosanoides com efeitos próinflamatórios. Dessa forma, os ácidos graxos da série $\omega-3$ passam a exercer um efeito protetor, impedindo a formação de eicosanoides da série par e seus efeitos prejudiciais (LEE \& LIP, 2003).

Diante deste processo de metabolização, a ingestão adequada das duas séries de ácidos graxos poli-insaturados, em proporções ideais garante o controle dos processos de coagulação e de inflamação (LOTTENBERG, 2009).

\section{Efeitos dos ácidos graxos $\omega-3$ sobre o sistema cardiovascular}

\section{Anti-inflamatório}

Acredita-se que seja por meio da competição entre os ácidos $\omega$-3 e $\omega-6$ e de suas consequências na produção de eicosanoides a razão para os vários efeitos positivos observados com a suplementação de ácidos graxos $\omega$-3, tais como diminuição da agregação plaquetária e alterações na função endotelial (RICHARDSON et al., 2011).

O maior fornecimento de fontes ricas em EPA e DHA substituirão em parte, os ácidos graxos $\omega-6$ e o $A A$ da membrana de células como plaquetas, eritrócitos, neutrófilos, monócitos e células hepáticas. Como resultado desta substituição haverá um decréscimo na produção de PGE2 e LTB4, que são indutores de quimiotaxia de leucócitos e inflamação, responsáveis pelo aumento da geração de espécies reativas de oxigênio, reguladores da produção de citocinas próinflamatórias e TXA2, potente agregador plaquetário e vasoconstritor. Serão estimulados principalmente a formação de LTB5, com efeitos menos ativos na indução de inflamação e quimiotaxia, promovendo condição mais vasodilatadora e menos agregante plaquetário (CASA NOVA \& MEDEIROS, 2011).

Além de produzir mediadores com efeitos antiinflamatórios, sabe-se que os ácidos graxos $\omega$-3 produzem mediadores envolvidos na produção de resolvinas e protectinas. Estas moléculas são capazes de reduzir a resposta inflamatória e têm propriedades imunomodulatórias, promovendo redução da migração dos neutrófilos e de citocinas pró-inflamatórias. As resolvinas da série $E$ são derivadas do EPA e as da série $D$ são produzidas a partir do DHA. Já as protectinas foram assim denominadas por se relacionarem às ações antiinflamatórias do DHA. Ambas atuam sobre macrófagos promovendo fagocitose de neutrófilos e a migração para os órgãos linfáticos, além disso, inibem a indução do fator de necrose tumoral- $\alpha$ (TNF- 
a) e interleucinas 1, 2 e 6. Atuam também sobre as plaquetas, bloqueando a agregação plaquetária por meio do bloqueio seletivo de receptores de tromboxanos. As resolvinas regulam ainda as moléculas de adesão da superfície celular, o que também reduz a migração de neutrófilos para o local da inflamação (BARBALHO et al., 2011; COLUSSI et al., 2014).

Já no ano de 2000 RIDKER et al. (2000) realizaram um estudo de acompanhamento e observaram que, em mulheres, níveis aumentados dos marcadores inflamatórios proteína C-reativa, amiloide A sérica, interleucina $6 \mathrm{e}$ molécula de adesão intercelular solúvel tipo 1 são preditivos de risco de eventos cardiovasculares futuros. Os resultados observados nesta pesquisa confirmam que, na mulher, os marcadores de inflamação são importantes preditores do risco de eventos cardiovasculares e que, a partir de uma perspectiva fisiopatológica, apoiam a hipótese de que a aterosclerose é, em parte, uma doença inflamatória.

LOPEZ-GARCIA et al. (2004) pesquisaram a relação entre o consumo de ácidos graxos $\omega-3$ (ALA, EPA, DHA) e as concentrações plasmáticas de biomarcadores inflamatórios e de ativação endotelial em mulheres aparentemente saudáveis. Os resultados mostraram que existe uma relação inversa com níveis de proteína C-reativa, que são $29 \%$ menores nas dietas com maiores concentrações de w-3. Também são observadas reduções de $23 \%$ de interleucina $6,10 \%$ de Eselectina, $7 \%$ de molécula solúvel de adesão intracelular e $8 \%$ de molécula solúvel de adesão vascular. Os autores sugeriram, portanto, que os ácidos graxos $\omega$-3 estão associados a níveis menores de biomarcadores da inflamação e função endotelial, e que seus efeitos podem auxiliar na prevenção de doenças cardiovasculares por meio da redução do processo inflamatório.

A relevância da ativação de biomarcadores inflamatórios e endoteliais no processo aterogênico foi sugerida por vários estudos com pacientes humanos. Assim, a utilização da mensuração de proteína C-reativa e outros marcadores inflamatórios pode aumentar a capacidade de identificar pacientes com alto risco de desenvolver eventos cardiovasculares futuros (BLAKE \& RIDKER, 2003).

Além da diminuição nos níveis de biomarcadores promovidos pela produção de eicosanoides, acredita-se que os ácidos $\omega-3$ podem, ainda, diminuir a inflamação e ativação endotelial devido à diminuição de espécies reativas de oxigênio. As múltiplas duplas ligações das cadeias hidrocarbônicas do $\omega-3$ permitem reações com espécies reativas de oxigênio. O peróxido de hidrogênio, por exemplo, ativa a expressão de moléculas de adesão e a quimiotaxia de leucócitos mediante estimulação de citocinas, portanto, se a produção é reduzida, reduz-se também a estimulação inflamatória (LOPEZ-GARCIA et al., 2004).

\section{Antiarrítmico}

A possibilidade de que os AGPI $\omega-3$ pudessem apresentar efeitos antiarrítmicos surgiu a partir de estudos comparando a incidência de infarto agudo do miocárdio em esquimós e dinamarqueses. Pesquisas antigas já mostraram que os esquimós apresentavam uma incidência consideravelmente menor de infarto agudo do miocárdio quando comparados com a população dinamarquesa. Acreditou-se então, que essa baixa incidência poderia se dever ao maior consumo de peixes e, por consequência, maior consumo de ácidos graxos $\omega$-3 (BANG et al., 1980).

Em revisão de literatura realizada por RICHARDISON et al. (2011) foi evidenciado que vários pesquisadores constataram que os AGPIs $\omega-3$ podem ser 
capazes de evitar a morte súbita, evitar arritmias ventriculares em pacientes que utilizam cardioversores implantados, reduzir a ocorrência de complexos ventriculares prematuros, reduzir a frequência cardíaca, aumentar a variabilidade da frequência cardíaca e prevenir a fibrilação atrial. Atualmente sabe-se que estes efeitos antiarrítmicos são mediados por alterações nos canais iônicos que alteram o potencial de ação dos cardiomiócitos e evitam que focos ectópicos provoquem episódios de arritmia (COLUSSI et al., 2014).

Nos cardiomiócitos, o potencial de ação é iniciado (fase 0) com a rápida despolarização, que é caracterizada pela abertura de canais de sódio (Na) e o rápido influxo destes íons para o interior da célula. Após a despolarização, inicia-se o processo de repolarização (fase 1), com a abertura abrupta de canais de $\mathrm{Na}$, devido a uma corrente externa de potássio $(\mathrm{K})$ transitória. Em seguida, ocorre a fase de platô (fase 2), com a abertura de canais de cálcio ( $\mathrm{Ca}$ ) voltagem-dependentes que promovem o influxo lento de $\mathrm{Ca}$ e $\mathrm{Na}$, que mantém a célula em um estado despolarizado, dando o tempo necessário para que ocorra a contração da fibra muscular. O último passo (fase 3 ) é a repolarização rápida, que se inicia com a entrada rápida de íons $\mathrm{K}, \mathrm{O}$ Ca é transportado para o interior do retículo sarcoplasmático e para fora da célula através de canais de Ca e as bombas de $\mathrm{Na} / \mathrm{K}$ retiram os íons $\mathrm{Na}$ da célula, além de promover o retorno dos íons de $\mathrm{K}$ para $\mathrm{o}$ interior da membrana, reestabelecendo assim a polaridade da membrana na fase de repouso (fase 4) (MILLER et al., 1996).

Os ácidos graxos $\omega$-3 promovem a inativação de canais de Na voltagemdependentes, inativação dos canais de $\mathrm{Ca}$ voltagem-dependentes (tipo L) e inativação dos canais de $\mathrm{K}$ que promovem a saída do $\mathrm{K}$ da célula. Essas alterações nos canais iônicos provocam mudanças na entrada e saída de íons levando a célula a um estado de hiperpolarização, que mantem a célula miocárdica na fase de platô por um tempo maior. Nesta fase, mesmo que surjam focos ectópicos a célula não iniciará um novo potencial, o que impede que uma arritmia seja desencadeada (COLUSSI et al., 2014).

Durante a isquemia, o potencial de repouso da membrana das células na fronteira da zona isquêmica sobe lentamente, caso uma dessas células atinjam um limiar de potencial de ação durante um ponto vulnerável do ciclo cardíaco (fase de repouso), uma arritmia poderá ser desencadeada. Na presença de $\omega-3$, com a inibição dos canais iônicos de $\mathrm{Na}, \mathrm{Ca}$ e $\mathrm{K}$ as células que desencadeariam arritmias não conseguem desenvolver potenciais de ação (RICHARDSON et al., 2011).

Atualmente, três hipóteses tentam explicar quais os mecanismos eletrofisiológicos celulares são responsáveis por esses efeitos antiarrítmicos. Em primeiro lugar, acredita-se que o $\omega-3$ seja capaz de modificar canais iônicos da membrana celular de cardiomiócitos, por conter sítios específicos de ligação que, se ocupados, podem alterar sua função. A segunda hipótese é que esses ácidos graxos podem afetar a fluidez da membrana e subsequentemente, a função do canal iônico. A terceira hipótese considera que mudanças na constituição da membrana ao redor dos canais iônicos alteram os seus efeitos, pois o comprimento hidrofóbico dos canais iônicos é muitas vezes menor do que a espessura da membrana dos fosfolipídeos. Considera-se que a inserção de $\omega-3$ na membrana em torno dos canais diminua a tensão reduzindo a curvatura em volta dos canais, modificando assim a sua função (RICHARDSON et al., 2011).

MATTHAN et al. (2005) realizaram uma meta-análise com o objetivo de avaliar o impacto da administração de ácidos graxos $\omega$-3 em modelos animais de arritmia. Foram incluídos 27 estudos que utilizaram modelos de arritmias induzidas 
por isquemia, isquemia e reperfusão, espontâneas e induzidas por estimulação elétrica. Os trabalhos selecionados incluíram suplementação alimentar (23 estudos) e por infusão (quatro estudos). Os resultados mostraram que os ácidos $\omega-3$, EPA e DHA, promoveram efeitos antiarrítmicos, demonstrados por sua capacidade em evitar episódios de taquicardia ventricular e fibrilação ventricular após isquemia. Entretanto, não relataram efeitos significativos sobre a incidência de morte súbita.

Uma série de estudos ao longo de 20 anos foi conduzida para tentar comprovar os efeitos antiarrítmicos dos ácidos graxos $\omega$-3. Em 1994, um estudo com cães mostrou que a infusão por via intravenosa de EPA e DHA impediu o desenvolvimento de fibrilação ventricular fatal após indução de isquemia em sete de oito cães tratados (BILLMAN et al., 1994).

Estudo subsequente realizado em 1999 mostrou que a infusão intravenosa de ALA, EPA e DHA produziram efeitos semelhantes na prevenção de arritmias ventriculares, enfatizando que a infusão intravenosa aumenta os níveis de $\omega$-3 livre rapidamente, diferente do que ocorreria por via oral, devido ao processo de metabolização ser mais lento. Foi neste momento que se suspeitou que esses ácidos graxos provocassem a supressão de canais de cálcio voltagem-dependentes e que talvez isso pudesse alterar a contratilidade cardíaca (BILLMAN et al., 1999).

Após 16 anos dos estudos iniciais, os pesquisadores realizaram estudos com a mesma espécie utilizando desta vez suplementação por via oral e, após quatro meses da indução de isquemia, realizaram avaliações in vivo e in vitro e concluíram que a suplementação oral aumenta as concentrações de ácidos graxos $\omega$-3 na membrana de eritrócitos, bem como no tecido cardíaco, e que essas concentrações se correlacionam com as doses ingeridas por via oral. Também concluíram que a suplementação não provoca alterações na contratilidade do miocárdio, mesmo ocorrendo modificações nos canais de cálcio (BILLMAN et al., 2010).

Por fim, em 2012, avaliando também a suplementação por via oral, concluíram que os ácidos graxos $\omega$-3 promoveram diminuição da frequência cardíaca em repouso e após exercício e aumentaram a variabilidade da frequência cardíaca. Esses fatores têm importância significativa para o efeito antiarrítmico, uma vez que as arritmias fatais se desenvolvem em frequências cardíacas elevadas (BILLMAN, 2012).

CALÒ et al. (2005) realizaram estudo para avaliar a eficácia e segurança do tratamento pré e pós-operatório com $\omega$-3 na prevenção da ocorrência de fibrilação atrial (FA) após cirurgia de revascularização do miocárdio. No grupo tratado com $\omega$-3, a frequência desta arritmia atrial no pós-operatório foi de $15,2 \%$ (12 de 79 pacientes) em comparação com 33,3\% do grupo controle (27 de 81 pacientes). Considerando os pacientes do grupo do $\omega-3,16,6 \%$ tiveram mais de um episódio de FA durante a internação, enquanto $18,5 \%$ dos pacientes do grupo controle apresentaram mais de um episódio. Os pesquisadores concluíram que o uso do $\omega$-3 reduziu a incidência de FA e diminuiu o tempo de internação após o procedimento cirúrgico. Neste estudo, o tratamento com AGPIs $\omega-3$ determinou uma maior redução do risco de desenvolvimento deste tipo de arritmia (54,4\%) quando comparado com a redução de risco observada com outros fármacos (43\% betabloqueadores, $54 \%$ sotalol e $39 \%$ amiodarona). 
As dislipidemias são um fator de risco importante para o desenvolvimento de doenças cardiovasculares, pois relacionam-se principalmente com a formação de placa aterosclerótica, formada inicialmente pela agressão ao endotélio vascular, promovida por fatores de risco como elevação de lipoproteínas aterogênicas (LDL, IDL, VLDL e remanescentes de quilomícrons). A disfunção endotelial promovida aumenta a permeabilidade da íntima vascular às lipoproteínas plasmáticas, favorecendo a retenção das mesmas no espaço subendotelial. O depósito de lipoproteínas na parede arterial é, portanto, um processo-chave no início da aterogênese. Sendo assim, qualquer aumento nas concentrações de lipoproteínas aterogênicas pode aumentar o risco de doenças cardiovasculares (SPOSITO et al, 2007).

Segundo a IV Diretriz Brasileira de Dislipidemias (SPOSITO et al., 2007) existe uma relação dose-dependente entre o consumo de ácidos graxos $\omega$-3 e os níveis de triglicerídeos. Estudos clínicos mostraram que a suplementação com AGPIs $\omega-3$ podem diminuir os níveis de triglicerídeos em até $25 \%$ a 30\%. O $\omega-3$ reduz a síntese hepática de triglicerídeos fazendo com que seus níveis no sangue diminuam.

Entretanto, o $\omega-3$ pode aumentar discretamente os níveis de HDL e LDL devido ao aumento da conversão de VLDL em LDL, ou por ativação de receptores que levam ao aumento da lipólise intravascular (CASA NOVA \& MEDEIROS, 2011).

O estudo de PAULETTO et al. (1996) compararam os efeitos a longo prazo sobre os fatores de risco cardiovascular de uma dieta à base de peixe comparada a uma dieta vegetariana em pessoas do mesmo grupo étnico. As concentrações de $\omega-3$ encontrados nos peixes ingeridos pelos pescadores chegaram a cerca de 3-5 gramas diariamente. A porcentagem de ácidos graxos $\omega-3$ no plasma foi significativamente maior no grupo de pescadores, ao passo que no grupo de vegetarianos as concentrações de $\omega-6$ foram maiores. Os níveis de colesterol plasmático e de triglicerídeos no grupo de pescadores foram inferiores ao grupo dos vegetarianos, com concentrações de lipoproteínas 37\% menores no primeiro grupo. Entretanto, o consumo de gorduras totais e de AGS foi mantida baixa, o que pode ter auxiliado nas quedas das concentrações de LDL, HDL e colesterol total, confirmando que o consumo de ácidos graxos $\omega-3$ gera um perfil de risco cardiovascular mais favorável.

MOURA et al. (2012) realizaram pesquisa com o objetivo de avaliar o efeito da suplementação de vitaminas (niacina e piridoxina) e da combinação de azeite de oliva e óleo de milho (ricos em ácidos graxos AGPIs $\omega-3$ ) no perfil lipídico de ratos. A combinação de azeite de oliva e óleo de milho na dieta reduziu colesterol hepático, lipídeos totais e triacilglicerídeos hepáticos, além de proporcionar maior excreção fecal de lipídeos.

BOSCH et al. (2012) realizaram investigações da suplementação com 1 grama de ácidos graxos $\omega-3$ em pacientes com risco de desenvolvimento de diabetes mellitus do tipo 2 e constataram que os níveis de triglicerídeos foram reduzidos em $14,5 \mathrm{mg} / \mathrm{dL}$ em relação aos pacientes tratados com placebo, entretanto não observaram efeitos significativos sobre os outros lipídeos.

Diante de inúmeros estudos, segundo OOl et al. (2015) o conhecimento sobre a importância da regulação do metabolismo das lipoproteínas e os efeitos do $\omega-3$ se tornam importantes, pois melhorando-se o perfil lipídico é possível atuar diminuindo um dos fatores de risco para o desenvolvimento das doenças cardiovasculares. 
Segundo a Sociedade Brasileira de Cardiologia, a hipertensão arterial é uma doença com alta prevalência e é considerado um dos principais fatores de risco para o desenvolvimento de doenças cardiovasculares (TAVARES et al., 2010). Os ácidos graxos $\omega$-3 têm mostrado efeitos benéficos sobre a hipertensão. Os mecanismos envolvidos na diminuição da pressão arterial parecem estar relacionados com a diminuição da produção de eicosanoides a partir do $\omega-6$, que possuem efeitos vasoconstritores, aliados ao aumento da produção de eicosanoides a partir dos $\omega-3$, com efeitos vasodilatadores (CASA NOVA \& MEDEIROS, 2011).

Os efeitos anti-hipertensivos do $\omega-3$ relacionam-se com os benefícios sobre a função vascular, pois essa classe de AGPI aumenta a liberação de óxido nítrico (NO), tromboxano A2 e prostaciclinas 12, que são capazes de promover relaxamento no músculo liso vascular. A incorporação de $\omega-3$ nas membranas plasmáticas das células altera sua fluidez, permeabilidade e função. Em consequência disso, é provável que a atividade de enzimas, afinidade de receptores e a capacidade de transporte das células aumentem, incluindo a síntese e liberação de NO (MORI, 2006).

PAULETTO et al. (1996) compararam os efeitos a longo prazo de uma dieta à base de peixe (ricos em $\omega$-3) com uma dieta vegetariana de pessoas de um mesmo grupo étnico. Em relação à concentração de lipídeos no plasma, a dieta com grande consumo de peixe tinha maiores níveis de ácidos graxos AGPI $\omega-3$ e menores níveis de $\omega-6$ quando comparados com a dieta vegetariana. Foram observadas pressões arteriais médias menores no grupo dos pescadores em relação ao grupo dos vegetarianos, confirmando mudanças favoráveis no perfil de risco cardiovascular.

Os estudos indicaram ainda que os efeitos anti-hipertensivos do DHA são mais significantes em relação ao EPA, neste caso o DHA também foi relacionado com a melhora na função endotelial e respostas vasoconstritoras reduzidas. A ação vasodilatadora dos $\omega-3$ também parece estar relacionada com a supressão da secreção de aldosterona, em resposta à inibição do hormônio adrenocorticotrófico (ACTH). Este efeito pode estar relacionado com mudanças no sinal intracelular de transdução, alterações na viscosidade do plasma ou a uma menor atividade da enzima conversora de angiotensina. A inibição desta enzima reduz a produção de angiotensina II, produzindo o relaxamento vascular e redução da secreção de aldosterona. Além disso, foi relatado que o DHA diminui a ocorrência de apoptose vascular do músculo liso, ajudando a prevenir a fibrose da parede vascular e o desenvolvimento de hipertensão secundária (CABO et al., 2012).

\section{CONSIDERAÇÕES FINAIS}

Os ácidos graxos são componentes importantes da alimentação, pois fazem parte da constituição dos lipídeos, compostos constituintes de diferentes estruturas do organismo, como por exemplo, das membranas celulares. Por isso, podem exercem grandes influencias nos processos inflamatórios e relacionados ao sistema cardiovascular.

Os ácidos graxos poli-insaturados da série $\omega$-3 são capazes de influenciar o perfil lipídico, a pressão arterial, a eletrofisiologia do coração e o processo inflamatório, atuando como cardioprotetor, ao contrário dos ácidos graxos saturados 
e trans, que sabidamente podem aumentar o risco de desenvolvimento de doenças cardiovasculares.

É importante salientar que a maioria dos estudos que envolvem os ácidos graxos se contrapõe e envolve incertezas. Isso pode se dever ao fato de que, com algumas exceções, trata-se de estudos epidemiológicos que avaliam hábitos alimentares ao longo do tempo, o que pode envolver fatores de complexa interação relacionados a estilos de vida. Grande parte dos artigos publicados expõem revisões ou meta-análises desses estudos epidemiológicos na tentativa de chegar a uma conclusão final.

Existem evidências de que mudanças no padrão de alimentação e suplementação com ácidos graxos específicos geram efeitos benéficos para 0 organismo e podem atuar como cardioprotetores, por isso continuam a merecem grande atenção e devem ser envolvidas em novas pesquisas.

\section{REFERÊNCIAS}

ADKINS, Y.; KELLEY, D. S. Mechanisms underlying the cardioprotective effects of omega-3 polyunsaturated fatty acids. The Journal Nutritional Biochemistry, v.21, p.781-792, 2010.

ALONSO, A.; RUIZ-GUTIERREZ, V.; MARTÍNEZ-GONZÁlEZ, M. A. Monounsaturated fatty acids, olive oil and blood pressure: epidemiology, clinical and experimental evidence. Public Health Nutrition, v.9, n.2, p.251-257, 2006.

BANG, H. O.; DYERBERG, J.; SINCLAIR, H. M. The composition of the eskimo food in north western Greenland. American Journal Clinical Nutrition, v.33, p.26572661, 1980.

BARBALHO, S. M.; BECHARA, M. D.; QUESADA, K. R.; GOULART, R. A. Papel dos ácidos graxos ômega 3 na resolução dos processos inflamatórios. Medicina, v.44, n.3, p.234-240, 2011.

BERTOLINO, C. N.; CASTRO, T. G.; SARTORELLI, D. S.; FERREIRA, S. R. G.; CARDOSO, M. A. Influência do consumo alimentar de ácidos graxos trans no perfil de lipídeos séricos em nipo-brasileiros de Bauru, São Paulo, Brasil. Caderno de Saúde Pública, v.22, n.2, p.357-364, 2006.

BILLMAN, G. E. Effect of dietary omega-3 polynsaturated fatty acids in heart rate ond heart rate variability in animals susceptible or resistant to ventricular fibrillation. Frontiers Physiology, v.3, n.71, p.1-10, 2012.

BILLMAN, G. E.; HALLAQ, H.; LEAF, A. Prevention of ischemia-induced ventricular fibrillation by $\omega 3$ fatty acids. Proceedings of the National Academy Sciences, v.91, p.4427-4430, 1994.

BILLMAN, G. E.; KANG, J. X.; LEAF, A. Prevention of sudden cardiac death by dietary pure $\omega-3$ polyunsaturated fatty acids in dogs. Circulation Journal, v.99, p.2452-2457, 1999.

BILLMAN, G. E.; NISHIJIMA, Y.; BELEVYCH, A. E.; TERENTYEV, D.; XU, Y.; HAIZLIP, K. M. et al. Effects of dietary omega-3 fatty acids on ventricular function in 
dog with healed myocardial infarctions: in vivo and in vitro studies. American Journal Heart Circulation and Physiology, v.298, p.1219-1228, 2010.

BLAKE, G. J.; RIDKER, P. M. C-reactive protein and other inflammatory risk markers in acute coronary syndromes. Journal American College of Cardiology, v.41, p.37S-42S, 2003.

BOSCH, J.; GERSTEIN, H. C.; DAGENAIS, G. R.; DÍAZ, D.; DYAL, L.; JUNG, H.; MAGGIONO, A. P.; PROBSTFIELD, J.; RAMACHANDRAN, A.; RIDDLE, M. C.; RYDÉN, L. E.; YUSUF, S. N-3 fatty acids and cardiovascular outcomes in patients with dysglycemia. The Nex England journal of medicine, v.367, p.309-318, 2012.

BROUWER, I. A.; WANDERS, A. J.; KATAM, M. B. Effect of animal and industrial trans fatty acids in HDL and LDL cholesterol levels in humans a quantitative review.

Plos One, v.5, n.3, p.9434-9442, 2010.

CABO, J.; ALONSO, R.; MATA, P. Omega-3 fatty acids and blood pressure. British Journal Nutrition, v.107, p.195-S200, 2012.

CALÒ, L.; BIANCONI, L.; COLIVICCHI, F.; LAMBERTI, F., LORICCHIO, M. L.; RUVO, E.; et al. N-3 fatty acids fot the prevention of atrial fibrillation after coronary artery bypass surgery. Journal of the American College of Cardiology, v.45, p.1723-1728, 2005.

CASA NOVA, M. A.; MEDEIROS, F. Recentes evidências sobre os ácidos graxos poli-insaturados da família ômega-3 na doença cardiovascular. Revista do Hospital Universitário Pedro Ernesto, v.1, n.11, p.74-80, 2011.

COLUSSI, G., CATENA, C.; SECHI, L. A. N-3 polyunsaturated fatty acids effects on the cardiometabolic syndrome and their role cardiovascular disease prevention: na update from the recente literature. Recent Advances in Cardiovascular Drug Discovery, v. 9, p.78-96, 2014.

EBBESSON, S. O. E.; VORUGANTI, S.; HIGGINS, B.; FABSITZ, R. R.; EBBESON, L. O.; LASTON, S.; HERRIS, W. S.; KENNISH, J.; UMANS, B. D.; WANG, H.; DEVEREUX, R. B.; OKIN, P. M.; WEISSMAN, N. J.; MACCLEUR, J. W.; UMANS, J. G.; HOWARD, B. V. Fatty acids linked to cardiovascular mortality are associated with risk factors. International Journal Circumpolar Health, v. 74, p.1-11, 2015.

GONZÁLEZ, F. H. D. Bioquímica clínica de lipídeos. In: GONZÁLEZ, F. H. D, DA SILVA, S. C. Introdução à bioquímica clínica veterinária. 2a ed. Porto Alegre: Editora da UFRGS; p.121-152.2006.

KASTORINI, C. M.; MILIONIS, H. J.; ESPOSITO, K.; GIUGLIANO, D.; GOUDEVENOS, J. Á.; PANAGIOTAKOS, D. B. The effect of mediterranean diet on metabolic syndrome and its componentes. Journal of American College of Cardiology, v.57, n.11, p.1299-1313, 2011. 
LEE, K. W.; LIP, G. Y. H. The role of omega-3 fatty acids in the secondary prevention of cardiovascular disease. QJM: An International Journal of Medicine, v.96, p.465480, 2003.

LEMAITRE, R. N.; KING, I. B.; MOZAFFARIAN, D.; SOTOODEHNIA, N.; REA, T. D.; KULLER, L. H.; TRACY, R. P.; SISCOVICK, D. S. Plasma phospholipid trans fatty acids, fatal ischemic heart disease, and sudden cardiac death in older adults: the cardiovascular health study. Circulation Journal, v.114, p.209-215, 2006.

LEMAITRE, R. N.; KING, I. B.; RAGHUNATHAN, T. E.; PEARCE, R. M.; WEINMAM, S.; KNOPP, R. H. et al. Cell membrane trans-fatty acids and the risk of primary cardiac arrest. Circulation Journal, v.105, p.697-701, 2001.

LOPEZ-GARCIA, E.; SCHULZE, M. B.; MANSON, J. E.; MEIGS, J. B.; ALBERT, C. M.; RIFAI, N. et al. Journal of Nutrition, v.134, p.1806-1811, 2004.

LOTTENBERG, A. M. P. Importância da gordura alimentar na prevenção e no controle de distúrbios metabólicos e da doença cardiovascular. Arquivos Brasileiros de Endocrinologia \& Metabologia, v.53, n.5, p.595-607, 2009.

MATTHAN, N. R.; JORDAN, H.; CHUNG, M.; LICHTENSTEIN, A. H.; LATHROP, D. A.; LAU, J. A systematic review and meta-analysis of the impact of $\omega-3$ fatty acids on selected arrhythmia outcomes in animal models. Metabolism Clinical and Experimental, v.54, p.1557-1565, 2005.

MENTE, A.; KONING, L.; SHANNON, H. S.; ANAND, S. S. A systematic review of the evidence supporting a causal link between dietary factors and coronary heart disease. Archive International Medicine, v.169, n.7, p.659-669, 2009.

MICHA, R.; MOZAFFARIAN, D. Satured fat and cardiometabolic risk factors, coronary heart disease, stroke, and diabetes: a fresh look at the evidence. Lipids, v.45, p.893-905, 2010.

MILLER, M. S.; TILLEY, L. P.; DETWEILER, D. K. Eletrofisiologia do coração. In: SWENSON, M. J.; REECE, W. O. Dukes Fisiologia dos animais domésticos. 11a ed. Rio de Janeiro: Editora Guanabara Koogan; p.95-132.1996.

MOREIRA, N. X.; CURI, R.; MANCINI FILHO, J. Ácidos graxos: uma revisão. Revista Nutrire, V.24, P.105-123, 2002.

MORI, T. A. Omega-3 fatty acids and hypertension in humans. Clinical and Experimental Pharmacology Physiology, v.33, p.842-846, 2006.

MOURA, F. A.; LAMEIRO, M. G. S.; TAVARES, R. A.; DIAS, A. R. G.; HELBIG, E.; BUCHWEITZ, M. R. D. Consumo de ácidos graxos mono e poli-insaturados e suplementação com niacina e piridoxina sobre e perfil lipídico de ratos wistar adultos. Alimentos e Nutrição, v.23, n.1, p.65-72, 2012. 
MOZAFFARIAN, P. WU, J. H. Y. Effects in risk factors, molecular pathways, and clinical events. Journal of American College of Cardiology, v.58, n.20, p.20472067, 2011.

NELSON, D. L.; COX, M. M. Lipídeos. In: NELSON, D.L.; COX, M. M. Princípios de bioquímica de Lehninger. 5a ed. Porto Alegre: Artmed; p.343-370.2011.

OOI, E. M. M.; WATTS, G. F.; NG, T. W. K.; BARRETT, H. P. H. R. Review effect of dietary fatty acids on human lipoprotein metabolism: a comprehensive update. Nutrients, v.7, p.4416-4425, 2015.

PAULETTO, P.; PUATO, M.; CAROLI, M. G.; CASIGLIA, E.; MUNHAMBO, A. E.; CAZZOLATO, G.; et al. Blood pressure and atherogenic lipoprotein profiles of fishdiet and vegetarian villagers in Tanzania: the Lugalawa study. Lancet, v.348, p.784788, 1996.

PIETINEN, P.; VARTIAINEM, E.; SEPPANEN, R.; ARO, A.; PUSKA, P. Changes in diet in finland from 1972 to 1992: impact on coronary heart disease risk. Preventive Medicine, v.25, p.243-250, 1996.

RAPOSO, H. F. Efeito dos ácidos graxos n-3 e n-6 na expressão de genes do metabolismo de lipídeos e risco de ateroesclerose. Revista de Nutrição, n.23, n.5, p.871-879, 2010.

RICHARDISON, E. S.; LAIZZO, P. A.; XIAO, Y. F. Electrophysiological mechanisms of the anti-arrythmic effects of omega-3 fatty acids. Journal Cardiovascular Translational Research, v.4, p.42-52, 2011.

RIDKER, P. M.; HENNEKENS, C. H.; BURING, J. E.; RIFAI, N. C-reactive protein and other markers on inflammation in the prediction of cardiovascular disease in women. The New England Journal of Medicine, v.342, p.836-843, 2000.

SANTOS, R. D.; GAGLIARDI, A. C. M.; XAVIER, H. T.; CASELLA FILHO, A.; ARAÚJO, D. B.; CESENA, F. Y.; ALVES, R. J. et al. Sociedade Brasileira de Cardiologia. I Diretriz Brasileira de Hipercolesterolemia Familiar (HF). Arquivos Brasileiros de Cardiologia, v.99, n.2 ,supl.2, p.1-28, 2012.

SANTOS, R. D.; GAGLIARDI, A. C. M.; XAVIER, H. T.; MAGNONI, C. D.; CASSANI, R.; LOTTENBERG, A. M. et al. Sociedade Brasileira de Cardiologia: I diretriz sobre o consumo de gorduras e saúde cardiovascular. Arquivos Brasileiros de Cardiologia, v.100, supl.3, p.1-40, 2013.

SPOSITO, A. C.; CARAMELLI, B.; FONSECA, F. A. H.; BERTOLAMI, M. C. Sociedade Brasileira de Cardiologia: IV diretriz brasileira sobre dislipidemias e prevenção de aterosclerose. Arquivos Brasileiros de Cardiologia, v.88, supl.1, p.1-19, 2007.

SUN, Q.; CAMPOS, H.; HANKINSON, S. E.; MANSON, J. E.; STAMPFER, M. J.; REXRODE, K. M.; WILLET, W. C.; HU, F. B. A prospective study of trans fatty acids 
in erythrocytes and risk of coronary heart disease. Circulation Journal, v.115, p.1858-1865, 2007.

TAVARES, A.; BRANDÃO, A. A.; SANJULTANI, A. F.; NOGUEIRA, A. R.; MACHADO, C. A.; POLI-DE-FIGEUIREDO, C. E. Sociedade Brasileira de Cardiologia/ Sociedade Brasileira de Hipertensão/ Sociedade Brasileira de Nefrologia: VI Diretrizes brasileiras de hipertensão. Arquivo Brasileiro de Cardiologia, v.95, supl.1, p.1-51, 2010.

VOET, D.; VOET, J. G. Lipídeos e membranas. In: VOET, D.; VOET, J. G. Bioquímica. 4a ed. Porto Alegre: Artmed; p.386-466.2013.

WHO. World Health Organization. Cardiovacular disease. Fact Sheet $n^{\circ} \quad 317$; 2013 [acesso 28 ago 2014]. Disponível em: http://www.who.int/cardiovascular_diseases/en/. 